\title{
Simplified Walking: A New Way to Generate Flexible Biped Patterns
}

\author{
Jinsu Liu ${ }^{1}$, Xiaoping Chen $^{1}$ and Manuela Veloso ${ }^{2}$ \\ ${ }^{1}$ Computer Science Department, University of Science and Technology of China, \\ Hefei, Anhui 230027, PRC \\ E-mail: jslau@mail.ustc.edu.cn xpchen@ustc.edu.cn \\ ${ }^{2}$ Computer Science Department, Carnegie Mellon University, \\ Pittsburgh, PA 15213, USA \\ E-mail: veloso@cmu.edu
}

\begin{abstract}
In this paper, we present a new way to generate biped patterns online. Linear Inverted Pendulum and Zero Moment Point are employed as the dynamic basics. The simplified walking concept is introduced to describe the complete biped motions with the unit of a walking step, instead of a time step of the robot's working time in usual methods. With this concept, the complex flexible motion planning can be realized with a low computation cost. We also propose the approach to design periodic biped motions and connect these motions together. The validity of our approach is demonstrated by experiments on simulated and real Nao robots.
\end{abstract}

\section{Introduction}

Flexibility is a key point in robot motion planning. How to change the motion of a biped robot smoothly and quickly is becoming an important topic. So far, there has been significant progresses focusing on flexible biped pattern generation. Nishiwaki et al. ${ }^{2}$ proposed an online method to connect subsequent patterns smoothly. ZMP preview control ${ }^{3}$ was introduced as a real-time approach to generate smooth CoM trajectory for given footsteps. The analytical solution based method ${ }^{4}$ was proposed to realize the stable and fast change of gait. The common feature of above approaches is that the ZMP concept ${ }^{1}$ is employed as the dynamic basic, and the CoM movement is presented by differential equations. Overall, the time granularity of the pattern generation is limited to one time step in robot's working time.

This paper presents a new approach, namely Simplified Walking, as an efficient way to generate biped patterns. The time granularity of the 
planning is extended to a complete walking step. This is achieved through a method of converting the continuous pattern generation to the decision of a single ZMP for each walking step. According to a simplified walking, the walking patterns for the real robot can be generated in real-time, while the computation cost to connect different motions together is highly reduced. The simplified walking brings us a neat and brief description of the CoM movements in biped motions, which makes it is easy for the robot to realize long distance planning and online replanning.

\section{Dynamic Models}

The Zero Moment Point ${ }^{1}$ (ZMP) is an important concept in biped walking. The ZMP is defined as a point on the floor where the sum of all the moments (due to gravity and inertial forces) equals zero. ${ }^{5}$ When the ZMP is within the convex hull of the contact points between the feet and the floor, the robot can walk in a stable manner. We use the 3D Linear Inverted Pendulum (3D-LIP) to describe the approximate movement of a biped walking when the robot is supporting its body on one leg. A 3D-LIP is an inverted pendulum which moves in a specific plane. Assuming the constraint plane is a horizontal plane and the current ZMP of the pendulum is at $\left(p_{x}, p_{y}\right)$, the physics model shown in Figure 1. can be described as follows:

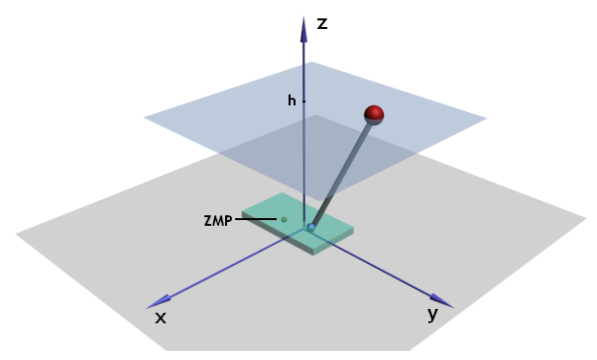

Fig. 1. 3D Linear Inverted Pendulum

$$
\begin{aligned}
& \ddot{x}=\frac{g}{h}\left(x-p_{x}\right) \\
& \ddot{y}=\frac{g}{h}\left(y-p_{y}\right)
\end{aligned}
$$

where $g$ is the acceleration due to gravity, $h$ is the given height of the constraint plane, $(x, y),(\ddot{x}, \ddot{y})$ are the position and the acceleration of the 
pendulum. Solving (1) as a differential equation, we get the relationship between a final state and an initial state in $\mathrm{x}$-axis of a LIP including the position and the velocity:

$$
\left[\begin{array}{c}
x_{f}(t) \\
v_{f}(t)
\end{array}\right]=A(t)\left[\begin{array}{c}
x_{i} \\
v_{i}
\end{array}\right]+[I-A(t)]\left[\begin{array}{c}
p_{x} \\
0
\end{array}\right]
$$

where $\left[x_{i}, v_{i}\right]^{T}$ is the initial state, $\left[x_{f}(t), v_{f}(t)\right]^{T}$ is the final state at time $t, \mathrm{I}$ is a $2 \times 2$ identity matrix, $A(t)=\left[\begin{array}{cc}\cosh (q t) & \frac{1}{q} \sinh (q t) \\ q \sinh (q t) & \cosh (q t)\end{array}\right]$ is a state transition matrix which only depends on the duration $t$, and $q$ is the constant $\sqrt{g / h}$. By defining the state as $X(t)=\left[\begin{array}{ll}x(t) & y(t) \\ \dot{x}(t) & \dot{y}(t)\end{array}\right]$ and the control input as $U=$ $\left[\begin{array}{cc}p_{x} & p_{y} \\ 0 & 0\end{array}\right]$, we obtain:

$$
X\left(t_{0}+t\right)=A(t) X\left(t_{0}\right)+[I-A(t)] U
$$

where $X\left(t_{0}\right)$ is the initial state of the LIP.

\section{Biped Pattern Generation Based on Simplified Walking}

Biped walking is a complex nonlinear dynamic process. It can be considered as a periodic phenomenon. A complete biped walking consists of two types of phases: ${ }^{5}$ the double-support phases and the single-support phases. Although the double-support phase is only about $20 \%$ of the duration of a walking cycle, it is important for making the walk more stable and reducing the impact between the swing foot and the ground. However, the single-support phase is the key part of biped walking. During this phase, the robot must place its swing foot in position for the next step while keeping its balance.

In this paper, we use a new approach, namely Simplified Walking, to generate biped walking patterns. Firstly, we simplify the walking process as a series of single-support phases acted on the CoM of the robot. Secondly, with the concept of simplified walking, we design periodic biped motions and connect them smoothly to achieve flexible walking. Finally, we convert a simplified walking to real patterns by considering double-support phases.

\subsection{Simplified Walking}

Simplified walking is specified as follows: 1) The robot is considered as a linear inverted pendulum which is supported by one of its feet. The mass 
of the robot is distributed on the Center of Mass (CoM) which moves in a horizontal plane with a constant height. The effects of inertia are ignored. 2) The walking process only consists of a series of single-support phases. Each single-support phase is named as a Simplified Step. The robot uses each of its legs as the supporting leg in turn. One simplified step is assumed to switch to the next one immediately. 3) While in a simplified step, we only use a single ZMP, namely ZMP Decision, instead of a ZMP trajectory in real biped walking.

To ensure the CoM trajectory is continuous and smooth, the final state of the $k$ th simplified step should have the same position and velocity as the initial state of the $k+1$ th step. The transition equation from the initial state of the $k$ th simplified step to the initial state of the $k+1$ th step is given by:

$$
X_{k+1}=A_{k} X_{k}+\left[I-A_{k}\right] U_{k}
$$

Therefore, the continuous CoM trajectory generation is converted to a discrete planning problem. We find a useful characteristic which is important for our walking planning.

Theorem 3.1. With two given supporting durations, there always and only exists a pair of ZMP decisions, which can be used for two simplified steps respectively, to make the LIP swing from an initial state to a final state.

Proof. We assume $p_{1}$ and $p_{2}$ are ZMP decisions applied on two simplified steps in turn. We obtain two state transition equations according to (5): $X_{m}=A_{1} X_{i}+\left(I-A_{1}\right) U_{1}$ and $X_{f}=A_{2} X_{m}+\left(I-A_{2}\right) U_{2}$, where $X_{i}$ is the initial state, $X_{f}$ is the final state, $X_{m}$ is the initial state of the second simplified step, $A_{1}$ and $A_{2}$ are the transition matrixes for these two steps respectively, $U_{1}=\left[\begin{array}{cc}p_{x 1} & p_{y 1} \\ 0 & 0\end{array}\right]$ and $U_{2}=\left[\begin{array}{cc}p_{x 2} & p_{y 2} \\ 0 & 0\end{array}\right]$ are the control inputs for these two steps. Since $|A(t)| \equiv 1$, transition matrix is invertible. Thus, we obtain $X_{m}=A_{2}^{-1} X_{f}+\left(I-A_{2}^{-1}\right) U_{2}$.

Let $E 1$ denote $\left[\begin{array}{ll}1 & 0 \\ 0 & 0\end{array}\right]$, let $E 2$ denote $\left[\begin{array}{ll}0 & 1 \\ 0 & 0\end{array}\right]$ and let $U$ denote $\left[\begin{array}{ll}p_{x 1} & p_{y 1} \\ p_{x 2} & p_{y 2}\end{array}\right]$. The control inputs can be written as $U_{1}=E_{1} U$ and $U_{2}=E_{2} U$. Then $U$ can be solved by $K U=A_{2}^{-1} X_{f}-A_{1} X_{i}$, where $K=\left(I-A_{1}\right) E_{1}-\left(I-A_{2}^{-1}\right) E_{2}$. Let $s_{i}$ and $c_{i}$ represent $\sinh \left(q t_{i}\right)$ and $\cosh \left(q t_{i}\right)$ respectively. According to the definition of transition matrix, we get $K=\left[\begin{array}{cc}1-c_{1} & c_{2}-1 \\ -q s_{1} & -q s_{2}\end{array}\right]$.

The durations $t_{i}$ are positive values, therefore $|K|=q\left(c_{1} s_{2}+c_{2} s_{1}-s_{2}-\right.$ $\left.s_{1}\right)>0 . U$ always has the certain solution: $K^{-1}\left(A_{2}^{-1} X_{f}-A_{1} X_{i}\right)$. 


\subsection{Periodic Biped Motion Design}

Periodic biped motions are most often used in applications of humanoid robot. We define periodic biped motions as the biped walking motions which consist of three types of steps: starting steps, walking steps and stopping steps. Each walking starts from a pair of starting steps and ends up with a pair of stopping steps. A continuous walking can be generated by repeating a pair of walking steps. Figure 2 demonstrates an eight-step walking motion by repeating the walking steps twice.

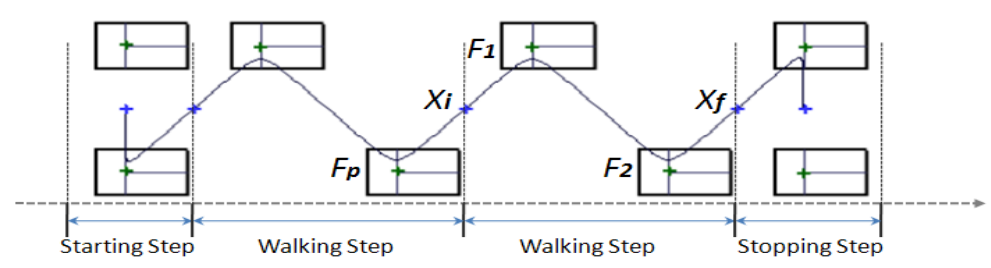

Fig. 2. Periodic biped motion

When we design a periodic biped motion, the landing gestures of the support foot are easy to be calculated according to the walking parameters including the step length, the turning angle and so on. To generate the CoM trajectory, we have to decide the state for each connecting point of different pairs of steps and calculate the ZMP decisions by using Theorem 3.1. For the purpose of making the walking steps can be executed repeatedly, the relative state of $X_{i}$ (shown in Figure 2) with respect to the previous support foot $F_{p}$ should be the same as the relative state of $X_{f}$ with respect to the support foot $F_{2}$. Therefore, the designment can be realized by choosing the proper relative state of $X_{f}$ with respect to $F_{2}$. The position of this relative state can be specified by walking parameters, while the velocity need to be tuned by considering wether the ZMP decisions calculated are within the stability margin $^{6}$ of the related support foot.

\subsection{Biped Motion Connection}

Biped motion connection is a technique to connect two simplified walking smoothly. Based on Theorem 3.1, the connection problem is defined as inserting two simplified steps in order to achieve the connection between the current walking and the connected walking.

The connection strategy is simple and efficient. We represent the current simplified walking as $\left(F_{1}, F_{2}, \ldots, F_{m}\right)$, where $F_{k}$ is the $k$ th simplified step, 
and the connected one as $\left(G_{1}, G_{2}, \ldots, G_{n}\right)$. When we desire the walking $\left(F_{1}, F_{2}, \ldots, F_{a}, G_{b}, \ldots, G_{n-1}, G_{n}\right)$, we modify the initial state of $G_{b}$ to have the same position as the final state of $F_{a}$, then we apply this modification onto all steps from $G_{b+1}$ to $G_{n}$. Two ZMP decisions can be calculated with the initial state of $F_{a}$ and the modified final state of $G_{b}$, then the ZMPs are used to generate the new CoM trajectory to achieve a smooth connection.

\subsection{Double-support Phase Generation}

With a series of ZMP decisions and related supporting durations, a smooth $\mathrm{CoM}$ trajectory can be generated. However, in a real walking process of a robot, the ZMP cannot be switched from one foot to the other immediately. We solved this problem by converting a part of each simplified step to double-support phase. The ZMP has a constant velocity in this phase in order to switch the ZMP smoothly.

We discretize the time with time step $\Delta t$. Let $x_{i}$ denote the position in $\mathrm{x}$-axis at the $i$ th time step. We present the acceleration at each time step as a difference equation: $\ddot{x}_{i}=\left(x_{i+1}+x_{i-1}-2 x_{i}\right) / \Delta t^{2}$. According to (1), the ZMP at each time step can be given by $p_{x_{i}}=x_{i}-\ddot{x}_{i} h / g$.

We introduce an evaluation to describe the difference between the current ZMP trajectory and the desired ZMP trajectory on the x-axis:

$$
E=\sum_{d_{1} \leq i \leq d_{2}}\left[p_{x_{i}}-p_{x_{i}}^{*}\right]^{2}
$$

where $p_{x_{i}}^{*}$ is the desired ZMP at the $i$ th time step, $d_{1}$ and $d_{2}$ are the beginning time step and the ending time step of this double-support phase. $x_{d_{1}-1}$ and $x_{d_{2}+1}$ are the boundary positions from the neighboring singlesupport phases. $x_{d_{1}}$ to $x_{d_{2}}$ are solved by the following partial differential equations to obtain the minimum value of $E$.

$$
\frac{\partial E}{\partial x_{i}}=0 \quad\left(d_{1} \leq i \leq d_{2}\right)
$$

Similarly, we can calculate the CoM trajectory on the y-axis to generate a complete double-support phase trajectory as shown in Figure 3.

\section{Experiments}

The humanoid robot $\mathrm{NAO}^{7}$ is used in our experiments. To demonstrate the biped motion connection, we conducted simulated experiments. Figure 4(a) shows a smooth connection of two CoM trajectories. The simulated robot achieved the connecting steps successfully as shown in Figure 4(b). 


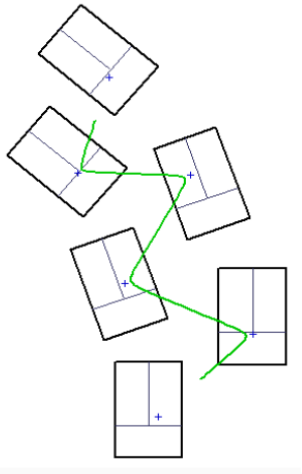

(a) Simplified Walking

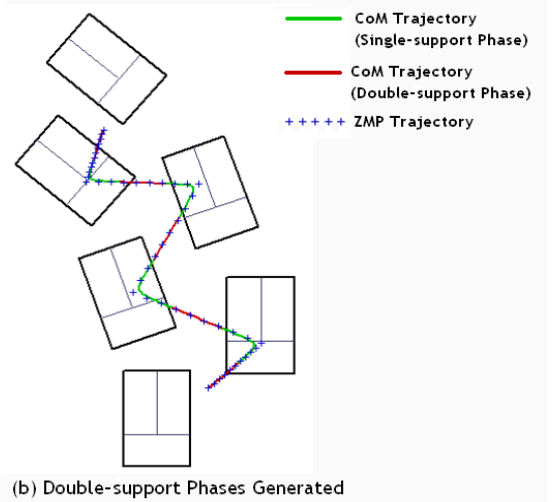

(b) Double-support Phases Generated

Fig. 3. Double-support phase generation

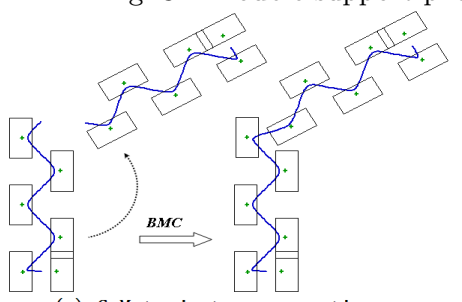

(a) CoM trajectory connection

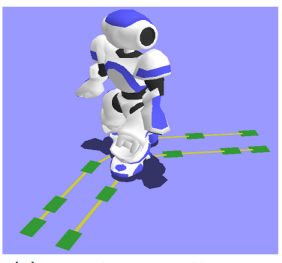

(b) Simulated wa1king

Fig. 4. Biped motion connection
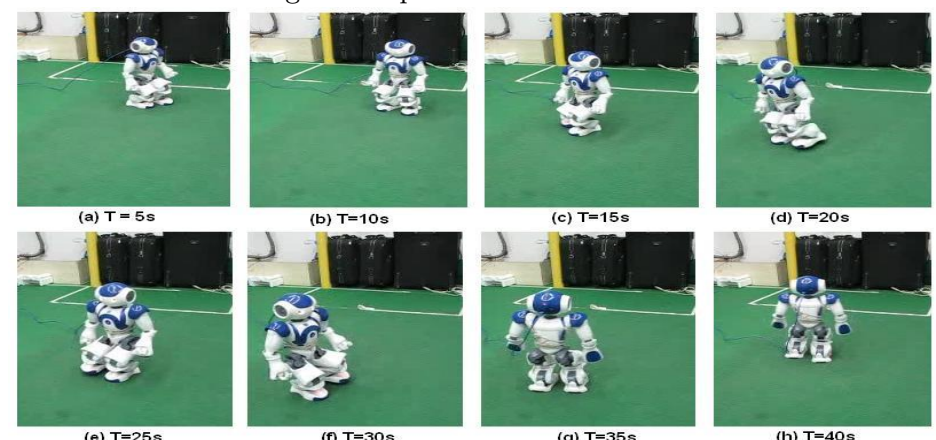

(f) $\mathrm{T}=30 \mathrm{~s}$

(g) $T=35 \mathrm{~s}$

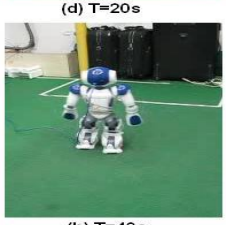

Fig. 5. Online biped motion planning controlled by a joystick

With the techniques mentioned in this paper, we designed several periodic biped motions including forward walking, sideways walking, turning and so on. We realized an online motion planner to generate walking patterns for a real Nao robot. The subsequent motion can be chosen by a joystick and connected to the current walking motion automatically. A flexible 
biped motion is demonstrated in the snapshots in Figure 5. Moreover, we have successfully used our simplified walking method in complicated tasks such as robot soccer competitions and gained the first place at the 2008 RoboCup China-Open. ${ }^{8}$

\section{Conclusions}

Comparing with the ZMP preview control, ${ }^{1}$ the simplified walking does not request the ZMP trajectory within a previewing period. A walking pattern does not have to be generated until the moment it will be achieved by the robot (except the CoM trajectory of a double-support phase is generated at one time). The robot is able to modify the landing gesture for the next step before landing the current swing foot. Therefore, by using simplified walking, we achieved the same flexibility as the analytical solution based method, ${ }^{4}$ but with much lower computation cost. In conclusion, the main advantage for our approach is that: the description of the trunk movement is presented by a series ZMP decisions instead of a complete CoM trajectory.

In the future, we will focus on seeking more intellectualized methods to design periodic biped motions. The dynamics model of simplified walking will be extended to describe the biped motions with alterable CoM height.

\section{Acknowledgements}

This research is supported by National Nature Science Foundation of China (Grant No. 60745002) and National 863 Programme (Grant No. 2008AA01Z150).

\section{References}

1. Vukobratovic, M. and Borovac, B., Zero-Moment Point - Thirty Five Years of Its Life, Int. Journal of Humanoid Robotics, Vol.1, No.1, 2004, pp. 157-173

2. Nishiwaki, K. and Kagami S. et al., Online Generation of Humanoid Walking Motion based on a Fast Generation Method of Motion Pattern that Follows Desired ZMP, in Proc. of the 2002 IEEE IROS, vel. 3, 2002, pp. 2684-2689

3. Kajita, S. and Kanehiro, F. et al., Biped Walking Pattern Generation by using Preview Control of Zero-Moment Point, ICRA, 2003, pp. 31-37

4. Haradam K. and Kajita, S. et al., An Analytical Method on Real-time Gait Planning fot a Humanoid Robot, in Proc. of IEEE-RAS/RSJ Int. Conf. on Humanoid Robotics, vol. 2, 2004, pp. 640-655

5. Huang, Q. and Yokoi, K. et al., Planning Walking Patterns for a Biped Robot, IEEE Trans. Robotics and Automation, vol. 17, 2001, pp. 280-289

6. Huang, Q. and Nakamura Y., Sensory Reflex Control for Humanoid Walking, IEEE Trans. Robotics, vol. 21, no. 5, 2005, pp. 977-984

7. http://www.aldebaran-robotics.com

8. http://ai.ustc.edu.cn/rco/rco08/index.php 